\title{
Asymmetry gap in the electronic band structure of bilayer graphene
}

\author{
Edward McCann \\ Department of Physics, Lancaster University, Lancaster, LA1 4YB, United Kingdom
}

\begin{abstract}
A tight binding model is used to calculate the band structure of bilayer graphene in the presence of a potential difference between the layers that opens a gap $\Delta$ between the conduction and valence bands. In particular, a self consistent Hartree approximation is used to describe imperfect screening of an external gate, employed primarily to control the density $n$ of electrons on the bilayer, resulting in a potential difference between the layers and a density dependent gap $\Delta(n)$. We discuss the influence of a finite asymmetry gap $\Delta(0)$ at zero excess density, caused by the screening of an additional transverse electric field, on observations of the quantum Hall effect.
\end{abstract}

PACS numbers: 73.63.-b, 71.70.Di, 73.43.Cd, 81.05.Uw

Recently there has been a huge amount of theoretical interest in the low energy electronic properties of ultrathin graphite films, including graphene monolayers [1, 2] and bilayers [3, 4]. This activity followed the successful fabrication of ultrathin graphite films [5] and subsequent measurements of an unusual sequencing of quantum Hall effect plateaus 6, 7]. While the Hamiltonian for low energy electrons in a monolayer describes Dirac-like chiral quasiparticles with Berry phase $\pi$ [1, 2], the low energy Hamiltonian for a bilayer describes chiral quasiparticles with a parabolic dispersion and Berry phase $2 \pi[3]$.

Theoretical [3] and experimental [8] studies of bilayer graphene have shown that asymmetry $\Delta=\epsilon_{2}-\epsilon_{1}$ between on-site energies $\epsilon_{1}, \epsilon_{2}$ in the layers leads to a gap between the otherwise degenerate conduction and valence bands as shown schematically in Fig. 1(a). Moreover, in contrast to graphene monolayers, there is an experimental possibility of controlling the magnitude of the gap $\Delta$ in the spectrum of bilayer graphene through the use of an external gate that is employed primarily to control the density of electrons $n$ on the bilayer system [5, 6, 7, 9]. Such a dependence $\Delta(n)$ owes its existence to the Coulomb interaction between electrons, but the density distribution itself is dependent on the value of $\Delta$ via the band structure. Here we use a self-consistent Hartree approximation to determine the electronic distribution on a bilayer in the presence of an external gate. The numerically calculated $\Delta(n)$ is shown in Fig. 11(b) (solid line) for typical experimental parameters in the case that the gap $\Delta=0$ at zero excess density [10]. It shows that the addition of density $n \sim 10^{12} \mathrm{~cm}^{-2}$ yields a gap $\Delta \sim 10 \mathrm{meV}$. The numerical curve is compared with the following analytic approximation (dashed line) valid for $\Delta \ll \gamma_{1}$ :

$$
\Delta \approx \frac{e^{2} L^{2} n}{2 C_{b}}\left[1+\frac{\Lambda \hbar^{2} v^{2} \pi|n|}{\gamma_{1}^{2}}-\Lambda \ln \left(\frac{\hbar v \sqrt{\pi|n|}}{\gamma_{1}}\right)\right]^{-1}
$$

where $C_{b}$ is the capacitance of a bilayer of area $L^{2}$, $v$ is the in-plane velocity of the bilayer and $\gamma_{1}$ is the inter-plane coupling. The dimensionless parameter $\Lambda=e^{2} L^{2} \gamma_{1} /\left(2 \pi \hbar^{2} v^{2} C_{b}\right)$ describes the effectiveness of the screening of the bilayer. The limit $\Lambda \rightarrow 0$ describes poor screening when the density on each layer is equal to $n / 2$

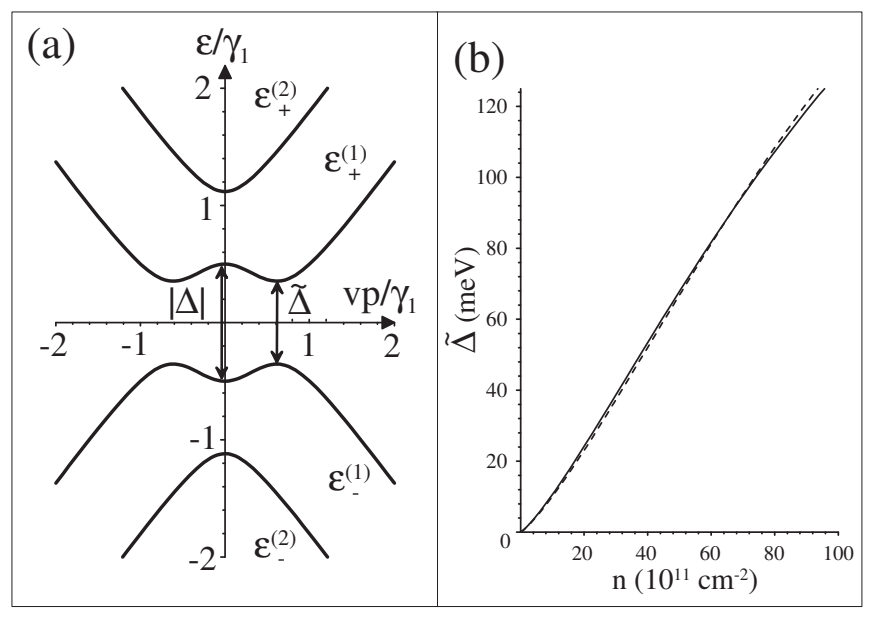

FIG. 1: (a) schematic of the electronic bands near the $K$ point in the presence of finite layer asymmetry $\Delta$ (for illustrative purposes a very large asymmetry $\Delta=\gamma_{1}$ is used), (b) numerically calculated dependence $\tilde{\Delta}(n)$ (solid line) compared with analytic expression (dashed line) using Eq. (11). For these values of density $\tilde{\Delta} \approx \Delta[10]$.

whereas for $\Lambda \rightarrow \infty$ there is excellent screening, the density lies solely on the layer closest to the external gate and $\Delta=0$. For typical experimental parameters [11] we estimate that $\Lambda \sim 1$.

We consider a graphene bilayer, with interlayer separation $c_{0}$, located a distance $d$ from a gate. An external gate voltage $V_{g}=e n d / \varepsilon_{r}^{\prime} \varepsilon_{0}$ induces a total excess density $n=n_{1}+n_{2}$ on the bilayer where $n_{1}\left(n_{2}\right)$ is the excess density on the layer closest to (furthest from) the gate. Here $\varepsilon_{0}$ is the permittivity of free space, $\varepsilon_{r}^{\prime}$ is the dielectric constant and $e$ is the electronic charge. Imperfect screening of the effective charge density en from the gate leads to an excess density $n_{2}$ on the layer furthest from the gate, with a corresponding change in potential energy $\Delta U_{2}=e^{2} n_{2} c_{0} / \varepsilon_{r} \varepsilon_{0}$ that determines the layer asymmetry

$$
\Delta(n)=\epsilon_{2}-\epsilon_{1} \equiv \Delta_{0}+e^{2} n_{2} L^{2} / C_{b},
$$

where $C_{b}=\varepsilon_{r} \varepsilon_{0} L^{2} / c_{0}$ and $\varepsilon_{r}$ is the bilayer dielectric constant. We introduce the bare asymmetry parameter $\Delta_{0}$ due to an additional transverse electric field pro- 
ducing finite asymmetry $\Delta(0)$ at zero density. The following analysis includes (a) the tight binding model of bilayer graphene including the asymmetry $\Delta$, (b) a selfconsistent determination of the gap numerically and, for $\epsilon_{F}, \gamma_{1} \gg \Delta$, analytically, and (c) a description of the influence of $\Delta_{0}$ on the cyclotron mass and the sequencing of quantum Hall effect plateaus at low density.

(a) The bilayer is modelled as two coupled hexagonal lattices with inequivalent sites $A 1, B 1$ and $A 2, B 2$ in the first and second graphene sheets, respectively, arranged according to Bernal (A2-B1) stacking. This lattice has a degeneracy point at each of two inequivalent corners, $K$ and $\tilde{K}$, of the hexagonal Brillouin zone which determine the centers of two valleys of a gapless spectrum. At the degeneracy point, electron states on inequivalent $(A 1 / B 1$ or $A 2 / B 2)$ sublattices in a single layer are decoupled, whereas interlayer coupling $\gamma_{A 2 B 1} \equiv \gamma_{1}$ forms dimers from pairs of $A 2-B 1$ orbitals in a bilayer, thus leading to the formation of high energy bands [3, 12]. The tight-binding Hamiltonian operates in the space of wave functions $\Psi=\left(\psi_{A 1}, \psi_{B 2}, \psi_{A 2}, \psi_{B 1}\right)$ in the valley $K$ and of $\Psi=\left(\psi_{B 2}, \psi_{A 1}, \psi_{B 1}, \psi_{A 2}\right)$ in the valley $\tilde{K}[3]$ :

$$
\mathcal{H}=\xi\left(\begin{array}{cccc}
-\frac{1}{2} \Delta & 0 & 0 & v \pi^{\dagger} \\
0 & \frac{1}{2} \Delta & v \pi & 0 \\
0 & v \pi^{\dagger} & \frac{1}{2} \Delta & \xi \gamma_{1} \\
v \pi & 0 & \xi \gamma_{1} & -\frac{1}{2} \Delta
\end{array}\right)
$$

where $\pi=p_{x}+i p_{y}$ and $\xi=+1(-1)$ labels valley $K(\tilde{K})$. The Hamiltonian takes into account asymmetry $\Delta=\epsilon_{2}-$ $\epsilon_{1}$ between on-site energies in the two layers, $\epsilon_{2}=\frac{1}{2} \Delta$, $\epsilon_{1}=-\frac{1}{2} \Delta$. Within each plane, nearest neighbor coupling as parameterised by $\gamma_{0}$ leads to in-plane velocity $v=$ $(\sqrt{3} / 2) a \gamma_{0} / \hbar$ where $a$ is the lattice constant.

At zero magnetic field, the Hamiltonian $\mathcal{H}$ describes four valley-degenerate bands, $\epsilon_{ \pm}^{(\alpha)}(\mathbf{p}), \alpha=1,2$, with

$\epsilon^{(\alpha) 2}=\frac{\gamma_{1}^{2}}{2}+\frac{\Delta^{2}}{4}+v^{2} p^{2}+(-1)^{\alpha} \sqrt{\frac{\gamma_{1}^{4}}{4}+v^{2} p^{2}\left(\gamma_{1}^{2}+\Delta^{2}\right)}$,

where $p$ is the magnitude of the momentum near the $K$ point, Fig. 1(a). The energies of the bands exactly at the $K$ point are $\left|\epsilon_{ \pm}^{(2)}(0)\right|=\sqrt{\gamma_{1}^{2}+\Delta^{2} / 4}$ and $\left|\epsilon_{ \pm}^{(1)}(0)\right|=$ $|\Delta| / 2$. Thus $\epsilon_{ \pm}^{(2)}$ describes higher-energy (A2-B1 dimer) bands $\left|\epsilon_{ \pm}^{(2)}\right| \geq \gamma_{1}$ whereas $\epsilon_{ \pm}^{(1)}$ are low energy bands split by the layer asymmetry $\Delta$ at the $K$ point [10].

The electronic densities $n_{1}$ and $n_{2}$ on the individual layers are given by an integral with respect to momentum over the circular Fermi surface $n_{1(2)}=\left(2 / \pi \hbar^{2}\right) \int p d p\left(\left|\psi_{A 1(2)}(p)\right|^{2}+\left|\psi_{B 1(2)}(p)\right|^{2}\right)$ where we have included a factor of four to take into account spin and valley degeneracy. On determining the wavefunction amplitudes on the four atomic sites we find

$$
\begin{aligned}
n_{1(2)} & =\int d p p g_{\mp}(\epsilon, p), \\
g_{\mp}(\epsilon, p) & =\frac{\epsilon \mp \Delta / 2}{\pi \hbar^{2} \epsilon}\left[\frac{\left(\epsilon^{2}-\Delta^{2} / 4\right)^{2} \mp 2 v^{2} p^{2} \epsilon \Delta-v^{4} p^{4}}{\left(\epsilon^{2}-\Delta^{2} / 4\right)^{2}+v^{2} p^{2} \Delta^{2}-v^{4} p^{4}}\right],
\end{aligned}
$$

where the minus (plus) sign is for the first (second) layer. The limits of integration are allowed values of momentum that, depending on the band in question and the value of the Fermi energy $\epsilon_{F}$, are $p=0$ or $p_{ \pm}$where $v^{2} p_{ \pm}^{2}=$ $\epsilon_{F}^{2}+\frac{1}{4} \Delta^{2} \pm\left[\epsilon_{F}^{2}\left(\gamma_{1}^{2}+\Delta^{2}\right)-\frac{1}{4} \gamma_{1}^{2} \Delta^{2}\right]^{1 / 2}$.

(b) The self-consistent calculation begins with zero external gate voltage, assuming the Fermi energy to be located at $\epsilon=0$. We take into account the contribution of the two valence bands to the initial densities on each layer, $n_{1}^{(0)}=-n_{2}^{(0)}$, and evaluate the gap $\Delta(0)$ using Eqs. (24). Then, we proceed to the case where an external gate voltage produces excess densities $n_{1}, n_{2}$, $n=n_{1}+n_{2} \neq 0$, and a gap $\Delta(n)$. Figure 2(a) shows $\Delta(n)$ for different values of the bare asymmetry $\Delta_{0}$ [10] and Fig. 2(b) shows the variation of the individual layer densities $n_{1}$ and $n_{2}$ for finite $\Delta_{0}$. The main effect of finite $\Delta_{0}$ (dashed and dotted lines in Fig. 2(a)) is to shift the plot $\Delta(n)$ with respect to the $\Delta_{0}=0$ case (solid line), but, as a result of screening, the asymmetry at zero density $\Delta(0)$ is smaller than the bare asymmetry $\Delta_{0}$. For example, the dotted curve for $\Delta_{0}=0.2 \gamma_{1}=78 \mathrm{meV}$ shows $\Delta(0) \approx 21 \mathrm{meV}$. Figure 2 (c) shows the variation of $\Delta(0)$ with bare asymmetry $\Delta_{0}$ (solid line).

We find an analytic expression for $\Delta(n)$ in the limit $\gamma_{1}, \epsilon_{F} \gg|\Delta|$ by evaluating the density on each layer Eq. (41) using an expansion in $\Delta / \gamma_{1}$. For simplicity, we consider $|\Delta| / 2<\left|\epsilon_{F}\right|<\gamma_{1}$ so that either the band just above or just below zero energy is partially occupied. On integrating Eq. (44) from zero momentum to $p_{F}=p_{+}$, we find the densities in the partially occupied bands. We also take into account the redistribution of density within the valence bands for $\epsilon_{F} \neq 0$ as compared to the $\epsilon_{F}=0$ case, as given by $\pm \gamma_{1} \Delta /\left(4 \pi \hbar^{2} v^{2}\right) \ln \left(4 \gamma_{1} /|\Delta|\right)$, so that

$n_{1(2)} \approx \frac{\operatorname{sgn}\left(\epsilon_{F}\right) p_{F}^{2}}{2 \pi \hbar^{2}} \pm \frac{\gamma_{1} \Delta}{2 \pi \hbar^{2} v^{2}}\left[\frac{\epsilon_{0}}{\gamma_{1}}+\frac{\epsilon_{0}^{2}}{\gamma_{1}^{2}}-\frac{1}{2} \ln \left(\frac{\epsilon_{0}}{\gamma_{1}}\right)\right]$,

where $\epsilon_{0}=\left(\gamma_{1} / 2\right)\left[\sqrt{1+4 v^{2} p_{F}^{2} / \gamma_{1}^{2}}-1\right]$ and $n=n_{1}+n_{2} \approx$ $\operatorname{sgn}\left(\epsilon_{F}\right) p_{F}^{2} /\left(\pi \hbar^{2}\right)$. If $|\Delta| \ll\left\{\left|\epsilon_{F}\right|, \gamma_{1}\right\}$ so that $v^{2} p_{F}^{2} \approx$ $\epsilon_{F}^{2}+\gamma_{1}\left|\epsilon_{F}\right|$, then $\epsilon_{0}$ is approximately independent of $\Delta$, and we may use Eq. (2) to find the gap,

$$
\Delta \approx \frac{\Delta_{0}+e^{2} L^{2} n /\left(2 C_{b}\right)}{1+\Lambda\left[\left(\epsilon_{0} / \gamma_{1}\right)+\left(\epsilon_{0} / \gamma_{1}\right)^{2}-\frac{1}{2} \ln \left(\epsilon_{0} / \gamma_{1}\right)\right]} .
$$

This is valid for intermediate densities $|\Delta|,\left|\Delta_{0}\right| \ll\left|\epsilon_{F}\right|<$ $\gamma_{1}$ where $\epsilon_{F} \approx \pm \epsilon_{0}$ [at very low density it incorrectly pre$\operatorname{dicts} \Delta(0)=0$ for $\left.\Delta_{0} \neq 0\right]$. In the regime $4 \pi \hbar^{2} v^{2}|n| \ll$ $\gamma_{1}^{2}$ and $\Delta_{0}=0$ it gives Eq. (1).

There is uncertainty in the value of the capacitance $C_{b}$ within this model because we assume that the excess charge is uniformly distributed within infinitesimally thin 2 d layers. Also, we used $\varepsilon_{r}=1$ for the dielectric constant of the bilayer which agrees with the prediction for other low dimensional structures 13], but is smaller than the value for bulk graphite $\varepsilon_{r} \approx 2.4$ [14]. Furthermore, we assume that the model parameters, including layer separation and interlayer coupling $\gamma_{1}$, do 


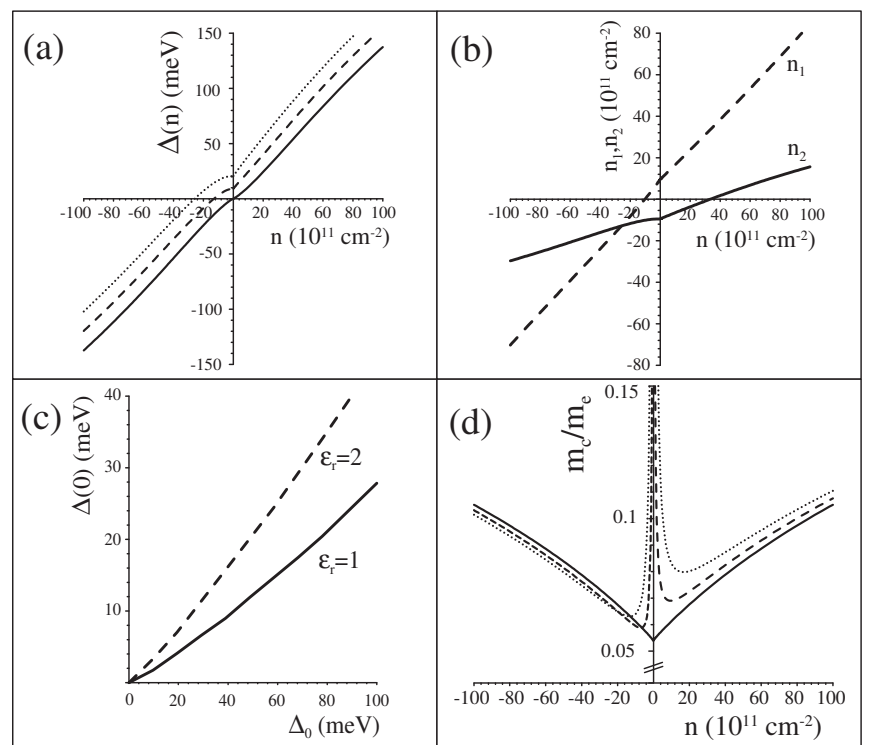

FIG. 2: Numerical evaluation of (a) the bilayer asymmetry $\Delta(n)$ for different values of the bare asymmetry $\Delta_{0}=0$ (solid line), $\Delta_{0}=0.1 \gamma_{1}=39 \mathrm{meV}$ (dashed line) and $\Delta_{0}=0.2 \gamma_{1}=$ $78 \mathrm{meV}$ (dotted line), using typical parameter values [11], (b) layer densities $n_{2}$ (solid) and $n_{1}$ (dashed) as a function of $n$ for $\Delta_{0}=0.2 \gamma_{1}=78 \mathrm{meV}$, (c) $\Delta(0)$ as a function of $\Delta_{0}$ for $\varepsilon_{r}=1$ (solid line) and $\varepsilon_{r}=2$ (dashed), and (d) the cyclotron mass $m_{c}$ in units of the bare electronic mass $m_{e}$, for different values of $\Delta_{0}$ as in (a).

not vary as a function of applied potential whereas a recent experiment [8] found that $\gamma_{1}$ changed by about $3 \%$ as $\Delta$ changed by $100 \mathrm{meV}$. The effectiveness of screening $\Lambda=e^{2} L^{2} \gamma_{1} /\left(2 \pi \hbar^{2} v^{2} C_{b}\right)$ depends on the model parameters as illustrated in Fig. 2(c) which shows $\Delta(0)$ as a function of $\Delta_{0}$ for $\varepsilon_{r}=1$ (solid line) and $\varepsilon_{r}=2$ (dashed): the gap $\Delta(0)$ increases with the dielectric constant.

(c) We use the self consistent analysis to evaluate the semiclassical expression for cyclotron mass $m_{c}$ that may be measured in an experimental observation at finite magnetic field of, say, Shubnikov de Haas oscillations [5, 6, 7]. For a circular Fermi surface, the semiclassical expression for cyclotron mass $m_{c}=p /(\partial \epsilon / \partial p)$ gives

$$
m_{c}=\frac{\left.\epsilon^{(\alpha}\right)}{v^{2}}\left[1+(-1)^{\alpha} \frac{\gamma_{1}^{2}+\Delta^{2}}{2 \sqrt{v^{2} p^{2}\left[\gamma_{1}^{2}+\Delta^{2}\right]+\gamma_{1}^{4} / 4}}\right]^{-1} .
$$

In the limit $\Delta=0$ for the low energy bands $\alpha=1$ this gives $m_{c}=\left(\gamma_{1} / 2 v^{2}\right) \sqrt{1+4 \pi \hbar^{2} v^{2}|n| / \gamma_{1}^{2}}$ [3]. Figure 2(d) shows the cyclotron mass $m_{c}$ as a function of total density $n$. For $\Delta_{0}=0$ (solid line) the mass is symmetric and finite $m_{c}=\gamma_{1} / 2 v^{2}$ at zero density. The other curves, for $\Delta_{0}>0$, show that the cyclotron mass for positive and negative densities is asymmetric with divergent behavior at low density resulting from the "mexican hat" structure of the low energy bands [12].

Another manifestation of finite $\Delta_{0}$ is in the sequencing of quantum Hall effect (QHE) plateaus at low density.
The Landau level (LL) spectrum of bilayer graphene has been discussed in Ref. [3]: here we briefly mention the features relevant for finite $\Delta_{0}$. The low energy states of electrons in bands $\epsilon_{ \pm}^{(1)}$ are conveniently described by an effective two component Hamiltonian that operates in the space of wave functions $\Psi=\left(\psi_{A 1}, \psi_{B 2}\right)$ in the valley $K$ and of $\Psi=\left(\psi_{B 2}, \psi_{A 1}\right)$ in the valley $\tilde{K}[3]$ :

$$
\begin{aligned}
\hat{H}= & -\frac{1}{2 m}\left(\begin{array}{cc}
0 & \left(\pi^{\dagger}\right)^{2} \\
\pi^{2} & 0
\end{array}\right) \\
& -\xi \Delta\left[\frac{1}{2}\left(\begin{array}{cc}
1 & 0 \\
0 & -1
\end{array}\right)-\frac{v^{2}}{\gamma_{1}^{2}}\left(\begin{array}{cc}
\pi^{\dagger} \pi & 0 \\
0 & -\pi \pi^{\dagger}
\end{array}\right)\right] \\
& -\frac{\alpha}{2 m}\left(\begin{array}{cc}
p^{2} & 0 \\
0 & p^{2}
\end{array}\right)+\frac{\beta \hbar e B}{2 m}\left(\begin{array}{cc}
1 & 0 \\
0 & -1
\end{array}\right)
\end{aligned}
$$

where $\pi=p_{x}+i p_{y}$ and $B$ is the magnetic field. For completeness we include the final two terms, with dimensionless parameters $|\alpha|,|\beta| \ll 1$, describing weak asymmetry between the conduction and valence bands. Neglected in Ref. [3], they arise due to additional couplings such as next-to-nearest neighbor in-plane coupling between $A 1$ $A 1$ and $B 2-B 2$ orbitals or interlayer coupling between $A 1-A 2$ and $B 1-B 2$ orbitals. The Landau level (LL) spectrum may be found using the Landau gauge $\mathbf{A}=(0, B x)$, in which operators $\pi^{\dagger}$ and $\pi$ coincide with raising and lowering operators [15] in the basis of Landau functions $e^{i k y} \phi_{N}(x)$, such that $\pi^{\dagger} \phi_{N}=i\left(\hbar / \lambda_{B}\right) \sqrt{2(N+1)} \phi_{N+1}$, $\pi \phi_{N}=-i\left(\hbar / \lambda_{B}\right) \sqrt{2 N} \phi_{N-1}$, and $\pi \phi_{0}=0$, where $\lambda_{B}=$ $\sqrt{\hbar /(e B)}$. The spectrum has almost equidistant energy levels for $N \geq 2$ with spacing $\hbar \omega_{c}, \omega_{c}=e B / m$, which are weakly split in valleys $K$ and $\tilde{K}(\xi= \pm 1)[\underline{3}]$,

$$
\epsilon_{N \geq 2}^{ \pm} \approx \frac{\xi \Delta \hbar \omega_{c}}{2 \gamma_{1}}-\alpha \hbar \omega_{c}\left(N-\frac{1}{2}\right) \pm \hbar \omega_{c} \sqrt{N(N-1)},(6)
$$

where $\epsilon_{N}^{+}$and $\epsilon_{N}^{-}$are assigned to electron and hole states, respectively, and we assume $|\Delta| / \gamma_{1} \ll 1,|\Delta| / \hbar \omega_{c} \lesssim 1$.

The LL spectrum in each valley also contains two levels identified using the fact that $\pi^{2} \phi_{1}=\pi^{2} \phi_{0}=0$,

$$
\begin{cases}\epsilon_{0}=-\frac{1}{2} \xi \Delta-\frac{1}{2}(\alpha-\beta) \hbar \omega_{c} ; & \Phi_{0 \xi} \equiv\left(\phi_{0}, 0\right) ; \\ \epsilon_{1}=-\frac{1}{2} \xi \Delta+\frac{\xi \Delta \hbar \omega_{c}}{\gamma_{1}}-\frac{1}{2}(3 \alpha-\beta) \hbar \omega_{c} ; & \Phi_{1 \xi} \equiv\left(\phi_{1}, 0\right) .\end{cases}
$$

According to different definitions of two-component $\Phi$ in two valleys, $N=0,1 \mathrm{LL}$ states in the valley $K$ are formed by orbitals predominantly on the $A$ sites from the bottom layer, whereas the corresponding states in the valley $\tilde{K}$ are located on $\tilde{B}$ sites from the top layer, as reflected by the splitting $\Delta$ between the lowest LL in the two valleys. The spectrum is shown on the right side of Fig. 3 assuming $|\Delta| / \gamma_{1} \ll 1,|\Delta| / \hbar \omega_{c} \lesssim 1$. In a symmetric bilayer $(\Delta=0)$ levels $\epsilon_{0}$ and $\epsilon_{1}$ are degenerate and have the same energy in valleys $K$ and $\tilde{K}$, thus forming an 8-fold degenerate LL at $\epsilon=0$ (here, spin is taken into account) as shown on the left side of Fig. 3 .

The LL spectrum is reflected in the QHE Hall conductivity dependence on carrier density, $\sigma_{x y}(n)$, although, 


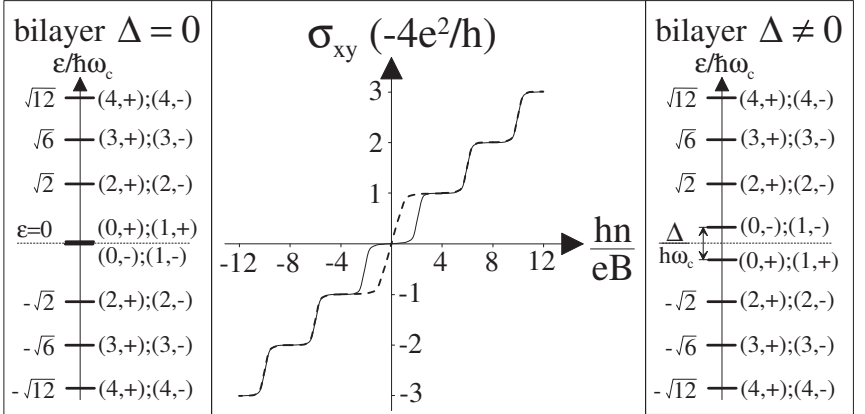

FIG. 3: Landau levels for zero (left) and finite asymmetry $\Delta$ (right). Brackets $(N, \xi)$ indicate LL number $N$ and valley index $\xi= \pm 1$. In the center the predicted Hall conductivity $\sigma_{x y}$ as a function of carrier density for bare asymmetry $\Delta_{0}=0$ (dashed line) is compared to that for $\Delta_{0} \neq 0$ (solid line).

in general, finite temperature and LL broadening tend to mask small LL splitting. When the asymmetry at zero density $\Delta(0)$, Fig. 2(c), is large enough that the percolating states 15 from the lowest levels in the two valleys can be resolved (the right side of Fig. 3), then these levels, embedded into the ladder of 4-fold degenerate LL's with $n \geq 2$, Eq. (6), result in the form of $\sigma_{x y}(n)$ as sketched in the center of Fig. 3 (solid line) which exhibits plateaus at all integer values of $4 e^{2} / h$ including a plateau at zero density. The temperature dependence of the zero density plateau will differ from that of the other plateaus because of the different activation energies, related to $\Delta(0)$ and $\hbar \omega_{c}$, respectively. When it is not possible to resolve the asymmetry splitting of the group of 8 states at $|\epsilon|=0$ (for $\Delta(0) \approx \Delta_{0} \approx 0$ ) then $\sigma_{x y}(n)$ (dashed line in Fig. 3) has plateaus at integer values of $4 e^{2} / h$ and a double $8 e^{2} / h$ step across $n=0$ 3, 7].

The use of doping 8] recently achieved gaps of up to about $200 \mathrm{meV}$. Alternatively, a potential difference of $30 \mathrm{~V}$ applied to a back gate placed $300 \mathrm{~nm}$ from the bilayer (combined with $-3 \mathrm{~V}$ applied to a top gate $30 \mathrm{~nm}$ away) translates into $30 \mathrm{mV}$ across the bilayer, $\Delta_{0}=30 \mathrm{meV}$, and $\Delta(0) \approx 7 \mathrm{meV}$. This compares with $\hbar \omega_{c} \approx 22 \mathrm{meV}$ for field $B=10 \mathrm{~T}[11]$ and level broadening due to scattering of approximately $3 \mathrm{meV}$ in recent experiments [9].

This paper has considered the influence of a single gate placed above an ultra thin graphite film as employed in recent experiments [5, 6, 7, 9] to control the density of electrons $n$ on the film. For the typical experimental range of densities shown in Fig. 1(b) the dependence of the asymmetry gap $\Delta(n)$ is roughly linear with $n$ with the addition of density $n \sim 10^{12} \mathrm{~cm}^{-2}$ yielding a gap $\Delta \sim 10 \mathrm{meV}$. Finite bilayer asymmetry gap $\Delta(0)$ at zero excess density, due to an additional transverse electric field, could influence observations of the integer quantum Hall effect by introducing a plateau at zero density in the Hall conductivity. This plateau is accompanied by a dip in the diagonal conductivity, the temperature dependence of which can be used to extract the value of the asymmetry gap $\Delta(0)$. This is distinguishable from the behavior of the higher plateaus with activation energies determined by the cyclotron frequency.

\section{Acknowledgments}

The author thanks V. I. Fal'ko, A. K. Geim and L. M. K. Vandersypen for useful discussions and EPSRC for financial support.
[1] D. P. DiVincenzo and E. J. Mele, Phys. Rev. B 29, 1685 (1984); T. Ando, T. Nakanishi, and R. Saito, J. Phys. Soc. Japan 67, 2857 (1998).

[2] Y. Zheng and T. Ando, Phys. Rev. B 65, 245420 (2002); V. P. Gusynin and S. G. Sharapov, Phys. Rev. Lett. 95, 146801 (2005); N. M. R. Peres, F. Guinea, and A. H. Castro Neto, Phys. Rev. B 73, 125411 (2006).

[3] E. McCann and V. I. Fal'ko, Phys. Rev. Lett. 96, 086805 (2006).

[4] J. Nilsson, A. H. Castro Neto, N. M. R. Peres, and F. Guinea, Phys. Rev. B 73, 214418 (2006); M. Koshino and T. Ando, Phys. Rev. B 73, 245403 (2006); J. Nilsson, A. H. Castro Neto, F. Guinea, and N. M. R. Peres, cond-mat/0604106; cond-mat/0607343. F. Guinea, A. H. Castro Neto, and N. M. R. Peres, Phys. Rev. B 73, 245426 (2006); M. I. Katsnelson, Eur. Phys. J. B 51, 157 (2006); 52, 151 (2006); B. Partoens and F. M. Peeters, Phys. Rev. B 74, 075404 (2006).

[5] K. S. Novoselov et al., Science 306, 666 (2004).

[6] K. S. Novoselov et al., Nature 438, 197 (2005); Y. Zhang et al., Nature 438, 201 (2005).

[7] K. S. Novoselov et al., Nature Physics 2, 177 (2006).

[8] T. Ohta, A. Bostwick, T. Seyller, K. Horn, and E. Roten- berg, Science 313, 951 (2006).

[9] Y. Zhang et al., Phys. Rev. Lett. 96, 136806 (2006).

[10] The true value of the gap between the conduction and valence band, occuring at $p \neq 0$, is $\tilde{\Delta}=|\Delta| \gamma_{1} /\left(\gamma_{1}^{2}+\right.$ $\left.\Delta^{2}\right)^{1 / 2}$. For huge asymmetry $|\Delta| \gg \gamma_{1}$, the gap saturates at $\tilde{\Delta} \approx \gamma_{1}$ although for modest asymmetry $|\Delta| \ll \gamma_{1}$, as considered throughout this paper, we have $\tilde{\Delta} \approx|\Delta|$.

[11] M.S. Dresselhaus and G. Dresselhaus, Adv. Phys. 51, 1 (2002); R.C. Tatar and S. Rabii, Phys. Rev. B 25, 4126 (1982); J.-C. Charlier, X. Gonze, and J.-P. Michenaud, Phys. Rev. B 43, 4579 (1991). We use $\gamma_{1}=0.39 \mathrm{eV}$, $c_{0}=3.35 \AA, \varepsilon_{r}=1$ and $v=8.0 \times 10^{5} \mathrm{~m} / \mathrm{s}[\underline{6}]$.

[12] We neglect the role of additional weak couplings, such as $A 1-B 2$ coupling $\gamma_{3}$ that results in trigonal warping and is relevant at low density $n \sim 1 \times 10^{11} \mathrm{~cm}^{-2}[\underline{3}]$.

[13] F. Léonard and J. Tersoff, Appl. Phys. Lett. 81, 4835 (2002).

[14] K. W.-K. Shung, Phys. Rev. B 34, 979 (1986); E. A. Taft and H. R. Philipp, Phys. Rev. 138, A197 (1965).

[15] "The Quantum Hall Effect", edited by R.E. Prange and S.M. Girvin (Springer-Verlag, New York, 1986). 